Controlling selective stimulations below a spinal cord hemisection using brain recordings with a neural interface system approach

This article has been downloaded from IOPscience. Please scroll down to see the full text article.

2011 J. Neural Eng. 8046008

(http://iopscience.iop.org/1741-2552/8/4/046008)

View the table of contents for this issue, or go to the journal homepage for more

Download details:

IP Address: 193.205.81.1

The article was downloaded on 31/05/2011 at 15:56

Please note that terms and conditions apply. 


\title{
Controlling selective stimulations below a spinal cord hemisection using brain recordings with a neural interface system approach
}

\author{
Fivos Panetsos $^{1,4}$, Abel Sanchez-Jimenez ${ }^{1}$, Carlos Torets ${ }^{1}$, Carla Largo ${ }^{1,2}$ \\ and Silvestro Micera ${ }^{3}$ \\ ${ }^{1}$ Neurocomputing and Neurorobotics Research Group, Universidad Complutense de Madrid, \\ 28037 Madrid, Spain \\ ${ }^{2}$ Experimental Surgery Department, La Paz Hospital, Madrid, Spain \\ ${ }^{3}$ ARTS Lab, Scuola Superiore Sant' Anna, Pisa, Italy \\ E-mail: fivos.panetsos@opt.ucm.es
}

Received 21 January 2011

Accepted for publication 27 April 2011

Published 31 May 2011

Online at stacks.iop.org/JNE/8/046008

\begin{abstract}
In this work we address the use of realtime cortical recordings for the generation of coherent, reliable and robust motor activity in spinal-lesioned animals through selective intraspinal microstimulation (ISMS). The spinal cord of adult rats was hemisectioned and groups of multielectrodes were implanted in both the central nervous system (CNS) and the spinal cord below the lesion level to establish a neural system interface (NSI). To test the reliability of this new NSI connection, highly repeatable neural responses recorded from the CNS were used as a pattern generator of an open-loop control strategy for selective ISMS of the spinal motoneurons. Our experimental procedure avoided the spontaneous non-controlled and non-repeatable neural activity that could have generated spurious ISMS and the consequent undesired muscle contractions. Combinations of complex CNS patterns generated precisely coordinated, reliable and robust motor actions.
\end{abstract}

(Some figures in this article are in colour only in the electronic version)

\section{Abbreviations}

CNS central nervous system

ISMS intraspinal microstimulation

NSI neural interface system

BMI brain-machine interface

Pr5 brainstem principal trigeminal nucleus

Tx $x$ th thoracic vertebra

Lx $x$ th lumbar vertebra

EMG electromyogram

4 Author to whom any correspondence should be addressed.

\section{Introduction}

Movement is the result of coordinated contractions of the muscles generated by the activity of neurons of the motor cortical areas. Electrical patterns are transmitted from the motor cortex to the peripheral muscles through a two-neuron pathway: the first neurons are the cells of the primary motor cortex; they send their axons to the second neurons located in the spinal cord; these second neurons send their axons to the peripheral muscles where they contact and stimulate individual muscle fibers [1]. Spinal cord injuries that damage the motor fibers interrupt the cortical input to spinal motoneurons below the lesion level and lead to paralysis of the muscles innervated by these neural cells. Since neurons 
in the CNS do not regenerate, spinal cord lesions always imply chronic paralysis for those affected. This represents a serious clinical and social problem that affects between 1.3 and 2.5 million people (250000-400 000 in the USA) as stated by the Christopher and Dana Reeve Foundation, the 2005 estimation of the International Campaign for Cures of Spinal Cord Injury Paralysis and the University of Alabama National Spinal Cord Injury Statistical Center [2-4]. According to the latter, a spinal cord injury implies average yearly expenses ranging from $\$ 228566$ to $\$ 775567$ in the first year and lifetime costs from $\$ 681843$ to over $\$ 3$ million for a 25 year old [4].

However, a permanent interrupt of the corticomedular connections does not lesion the motor cortex or the motoneurons of the spinal cord, but leaves both of them functionally active for many years after the lesion [5-8]. Therefore, they could be involved in bioengineering approaches for motor restoration and rehabilitation of paraplegic patients [9-11]. On one hand electrical microstimulation approaches have proven to be very useful for novel neurorehabilitation approaches: ISMS of the lumbosacral spinal cord can be helpful for the restoration of standing and walking $[12,13]$; it has been proven that cortical signals can be used for functional stimulation of the muscular system [14-16] and it has also been shown that epidural electrical stimulation of the dorsal columns restores locomotion in both acute pharmacologically induced dopamine-depleted mice and chronic six hydroxydopaminelesioned rats [17-19]. Recent advances in NSIs and BMIs show that specific devices for an electronic connection between cortical and spinal systems could be feasible in the very near future $[9,11,18-21]$. BMIs have been effectively controlled using very small numbers of neurons (never more than a few tens) and trained to generate the desired actions of the neuroprostheses.

Future devices for restoration of motor functions in paraplegic subjects will record the electrical activity of neurons in the cortical motor areas and generate the stimuli to activate spinal motoneurons $[5,14,22,23]$. It is then expected that neural plasticity should contribute to the achievement of a better control of voluntary movements as in the case of brachial plexus operations [24]. The same plasticity could also be useful to adapt CNS responses to the variability of the recordings since no multielectrode recordings have currently been fully verified to stably and reliably record action potentials from multiple single units for extended periods of time [9].

Although very significant, the above results only represent the starting point for an artificial corticospinal control to restore the motor capabilities of spinal patients. Important open questions in this context include:

(1) guarantee the robustness of the movements, and therefore the generation of stable ISMS from neural recordings of variable activity;

(2) guarantee the reliability of the movements, that means the repeatability of motor actions elicited by the recordings of identical neural patterns and;

(3) to determine procedures for suitable CNS neural activity patterns to be recorded and used for the generation of complex movements as well as the time relations between them and;

(4) to generate accurate muscle movements using a minimum number of neural recordings.

In this paper, we address the above questions in the case of NSI-recorded realtime CNS activity patterns and the generation of precise and coordinated muscle activity through ISMS.

We prove that CNS recordings and selective stimulation of the spinal motoneurons can produce fine, reliable and robust complex movements. We addressed the robustness of the movements by introducing variability in both the neural sampling and the spinal cord loci for the ISMS, and studying the robustness of the generation of precise movements. The reliability of CNS-triggered fiber contractions was tested by analyzing the variability of the EMGs under repetition of the same CNS pattern. By determining the time intervals of useful CNS activity, the location of the recording and stimulating electrodes, and the inclusion of delays between stimuli, we determined an experimental procedure for the generation of complex movements.

The rationale of our work has been the following: first, to record precise CNS neural activity patterns in a very selective manner and generate artificial electrical signals depending on the recorded patterns. After that, to employ these artificially generated signals to perform an open-loop differential functional stimulation of the spinal motoneurons (ISMS) to generate reliable and robust synchronous muscular activity. At this point, our study was centered in (i) combining the above neural patterns to generate complex movements as sequences of contractions of specific muscle fibers; (ii) studying the variability of the movements elicited by the recordings of identical neural patterns; and (iii) studying the stability of the movements by introducing noise through the generation of the ISMS.

However, due to their conscious nature, the cortical motor patterns necessary for our study cannot be generated in anesthetized animals, controlled, and repeated with precision by the researchers. Consequently, to investigate the precision and fine adjustment of coordinated muscle contractions, we have to move from the motor areas to some other locus, where the trigger of such neural activity patterns can be realized in a precise and repeatable manner. The best candidates are sensory systems (any) since peripheral stimulations generate repeatable responses to their neurons (visual, tactile, auditory, etc), and neural spikes are roughly similar in the sensory and motor pathways [1].

\section{Experimental procedure}

For our experiments, we chose the trigeminal tactile system of the rat, due to its highly topographic organization and the broad knowledge gathered on its anatomy and physiology throughout the last few decades [25-27]. This system is based on a tactile apparatus of a matrix of 33 long hairs (vibrissae or whiskers) located in the snout of the animal (figure 1, top left) with which the rat touches and perceives external objects. Sensory information from the whiskers is conveyed to the CNS in a 
(A)
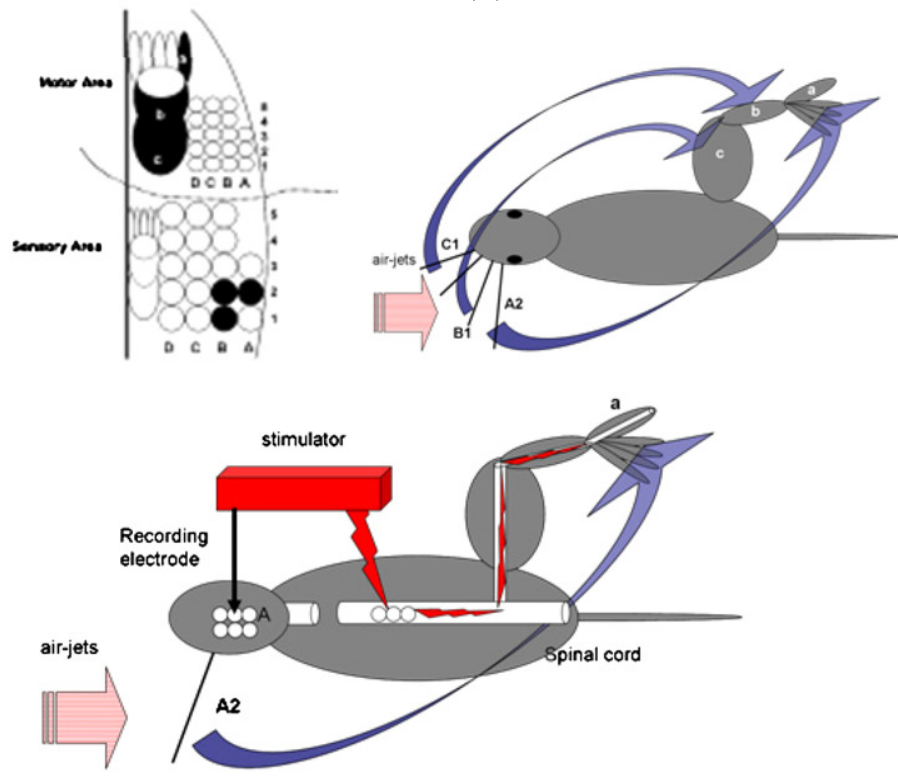

(B)

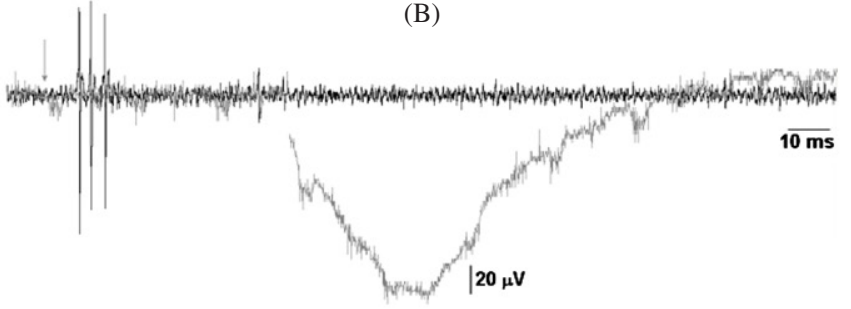

Figure 1. (A) Rationale of the experimental conditions. Top left: schematic presentation of the 'ratunculus', the somatotopically organized groups of neurons devoted to processing information related to such areas in the motor and sensory brain regions. Air-jet delivery to each of the vibrissae A2, B1, B2, C1 evokes neural activity in the corresponding brain regions of the sensory area (top right, black circles). Consider a tetrode placed in the sensory area, whose electrode one is recording from region A2, electrodes two and three from B1 and electrode four from B2. Air-jet stimuli delivered to the vibrissa A2 will evoke neural responses in region A2 that will be recorded by electrode one and used to generate stimulation patterns. These in turn are sent to the motoneurons of the spinal cord (bottom), which are being stimulated and generate movement of the digit muscles, with the same occuring for the other two vibrissae. The precise location of the stimulating electrodes was determined by an electrolytic lesion made at the end of the experiment. Note that this is only a conceptual scheme; it does not reflect the real topology, nor the proportions of the real nervous system. Indeed, rats have much more vibrissae than those depicted here. (B) CNS neural activity and sensory feedback of the elicited motor responses. Top: superimposed recordings from Pr5 (black) and muscle fibers (gray). Vibrissa stimulation (gray arrow) elicits a neural response of three spikes, which is recorded by us and transformed into an electrical stimulus. This stimulus provokes a contraction of the muscular fibers if applied to the spinal motoneurons (ISMS) corresponding to the sciatic nerve, as seen in the EMG signal. Neural activity and EMG are represented with different timescales (horizontal line corresponds to $10 \mathrm{~ms}$ for the EMG and $20 \mathrm{~ms}$ for the neural recording). Bottom: EMGs corresponding to muscle contractions at increasing amplitudes of the ISMSs (right). All EMGs were triggered by the same CNS electrical pattern (left, up) whose peristimuli histograms are shown at the bottom. Muscle contraction increases with the amplitude of the stimulus. Arrows indicate the onset of the stimuli.

very well organized manner. Here, groups of neurons that are spatially distributed in the same manner as the whiskers in the nose of the rat (somatotopic maps) respond to the movements of these hairs (figure 1, top right). The system is discrete and each group of neurons corresponds to only one vibrissa in the snout. Using this discrete somatotopic organization, we decided to assign to each vibrissa the control of a specific muscle fiber. Since the displacement of each vibrissa elicits neural responses in the corresponding map of the CNS, we planned to place microelectrodes in the CNS (Pr5 [25]) to record such responses. Since the displacement of each vibrissa elicits neural responses in the corresponding map of the CNS, to record such responses we put electrodes in the Pr5 [25] of the CNS. The coordinated displacements of different vibrissae generated a cascade of Pr5 neural responses and consequently the triggering of corresponding ISMS leading to the development of complex movements.

This way, instead of introducing our recording electrodes into the motor cortex and awaiting the appearance of spontaneous neural activity, by placing our electrodes into the somatosensory tactile system of the rat we should be able to trigger the desired neural patterns and repeat them in a very reliable manner. In addition, we could add bias/variability in the recorded patterns through precisely quantified variations in the stimulation parameters of the whiskers. Therefore, the entire detailed procedure, as depicted in a schematic manner in figure 1 and described with an example in the legend, consists of eight steps:

(i) introduction of multielectrode probes in Pr5;

(ii) recordings from at least three individual electrodes, each of which responds to the stimulation of a different vibrissa;

(iii) hemisection of the spinal cord at T11-L1 level (11th thoracic to first lumbar vertebra) to interrupt the corticospinal pathway;

(iv) introduction of three to four microelectrodes to the sectioned spinal cord caudally to the lesion, at level L3-L4 (third lumbar, fourth lumbar vertebra);

(v) identification of ISMS loci in which at least three individual electrodes generated contractions of different muscle fibers; 
(vi) stimulation of the rat vibrissae, recording, online processing of the neural activity and generation of ISMS patterns;

(vii) adjustment of the stimulation parameters to obtain selective non-overlapping contraction of small groups of muscle fibers and;

(viii) recordings of the muscular activity to obtain a sensory feedback.

Complex patterns of CNS activity and, consequently, patterns of movements were generated by combining stimulations of two to three vibrissae.

\section{Materials and methods}

Data were obtained from 23 urethane-anesthetized $\left(1.5 \mathrm{~g} \mathrm{~kg}^{-1}\right.$ i.p.) adult albino Wistar rats of both sexes, weighing 200$250 \mathrm{~g}$, mounted in the stereotaxic device with anesthesia, general surgical procedure and care (recordings and sensory stimulations as described in [27]). For the laminectomy, the skin was removed to discover the vertebrae from T10 to L5 and the spinal cord was exposed. The interrupt of the efferent fibers was realized at the level of T11-L1 using a microsurgery blaster under direct observation through a dissection microscope. Drying of the exposed surface was prevented by covering it with Vaseline oil. For recordings and stimulations, we used $0.5-1.0 \mathrm{M} \Omega$ tetrodes manufactured and tested in our lab using 30-125 $\mu \mathrm{m}$ diameter thread tungsten wires with a quadruple coating of Teflon following the Neuralynx methodology (www.neuralynx.com), or WPI tungsten microelectrodes 1.0-2.0 $\mathrm{M} \Omega$. For data acquisition, we used a Micro1401 mkII unit with accompanying Spike2 software (Cambridge Electronic Devices) and a PCI-6071E data acquisition card from National Instruments with the Recorder software amplified and displayed on a Plexon Inc. PCI device. Spikes were sampled and digitalized at $20 \mathrm{kHz}$, and analyzed online for the generation of the stimulation patterns using a threshold-based spike sorting procedure. They were also stored in personal computers for subsequent off-line analysis. Local field potentials, EEG and synchronization pulses were recorded at a frequency of $1.0 \mathrm{kHz}$.

For the extracellular recordings, multielectrode probes were directed vertically into the rat brain [27]. The location of the recording electrodes was inferred from the stereotaxic coordinates and from the somatotopy of the evoked responses [27]. For the stimulations, other electrodes were introduced into the spinal cord at the L3-L4 level. Spinal motoneurons were stimulated by current injection $(1-300 \mu \mathrm{A}, 100 \mu \mathrm{s})$ by means of SIUs (World Instruments Precision, A365 and A360 Stimulus Isolator Units) controlled by Spike software through the Micro1401 mkII data acquisition unit. EMGs were obtained with $2 \mathrm{~mm}$ long stainless-steel, intramuscular electrodes with a $40-50 \mu \mathrm{m}$ tip, referred to a similar electrode placed in an adjacent denervated muscle [28]. They were thrust into the muscle perpendicularly to its long axis in a location determined in preliminary experiments. All experiments were carried out according to EU directives (86/609/EC) and national legislation (RD 1201/2005) regarding this matter.
Precise and repeatable brain activity-generated movements were obtained from the responses of Pr5 neurons to air-jet stimuli delivered to the whiskers. The spikes generated by an individual whisker, integrated in a short time window $(100 \mathrm{~ms})$ after the appearance of the first spike, were used to control current delivery from one individual ISMS electrode. This way, ISMS precision directly depends on the neural activity; however, the strength of the contractions depends only on the intensity of the injected current. The amplitude of the time window (100 ms) was determined in order to reduce the probability of a random appearance of a spike to be $<10^{-5}$, according to the spontaneous activity of the nucleus [29]. Neural responses were evoked by delivering $1.0 \mathrm{~Hz}$ air-jet stimuli (10 psi, Picospritzer III, Parker Institute, TX, USA) to the whiskers of the rat in a rostrocaudal direction via a silicon tube, $0.5 \mathrm{~mm}$ in diameter, positioned at a distance of $10-12 \mathrm{~mm}$ from the vibrissa. Recordings from neurons responding to a single whisker were obtained by moving the electrodes along the dorsoventral axis while stimulating the vibrissae [27].

The precise location of the stimulating electrodes was determined by the histology of the spinal cord performed from $2 \mathrm{~mm}$ rostrally to the medullar lesion, to $2 \mathrm{~mm}$ caudally, to the most caudally placed stimulation electrode. To assess the extension of the medullar lesions as well as to identify the precise cerebral structures recorded and stimulated by the electrodes, brain waves were studied histologically. ISMS loci were determined by inducing electrolytic lesions passing 3-5 mA, $10 \mathrm{~s}$ long currents through the tip of the electrode at the end of the experiment. Animals were then injected with an overdose of sodium pentobarbital $\left(50 \mathrm{mg} \mathrm{kg}^{-1}\right)$ and perfused transcardially with saline followed by formalin ( $4 \%$ in saline). The brain was removed, stored in $20 \%$ sucrose saline and cut on a freezing microtome into $50 \mu \mathrm{m}$ coronal sections that were then stained using the Nissl method or reacted for cytochrome oxidase ( $\mathrm{CyO})$.

Selective phasic stimulation of the spinal motoneurons by the application of low intensity currents $(25-40 \mu \mathrm{A})$ through individual microelectrodes generated single muscle contractions of the hindlimb of the rats with complete hemisection of the spinal cord. Fiber contractions have been visually observed and documented by EMG and video recordings (see complementary audiovisual material). Current intensities higher than $50 \mu \mathrm{A}$ normally activated a large number of muscle fibers. Following the somatotopic organization of the medullar motoneurons, proximal and distal muscle fibers could be identified and stimulated in a reliable manner. Sequences of movements were obtained by combined micro stimulations of two to three microwires placed alongside the rostrocaudal axis at distances of $0.5-0.7 \mathrm{~mm}$ from each other. Stimulation thresholds for the generation of movements $(32 \pm 1.6 \mu \mathrm{A})$ remained constant during the course of the experiments. The evoked hindlimb movements also remained constant throughout.

\section{Results}

CNS activity elicited very precise repeated ISMS (99.2\%, variance $\left.<10^{-2} \mathrm{~ms}\right)$. In turn, they generated fiber 

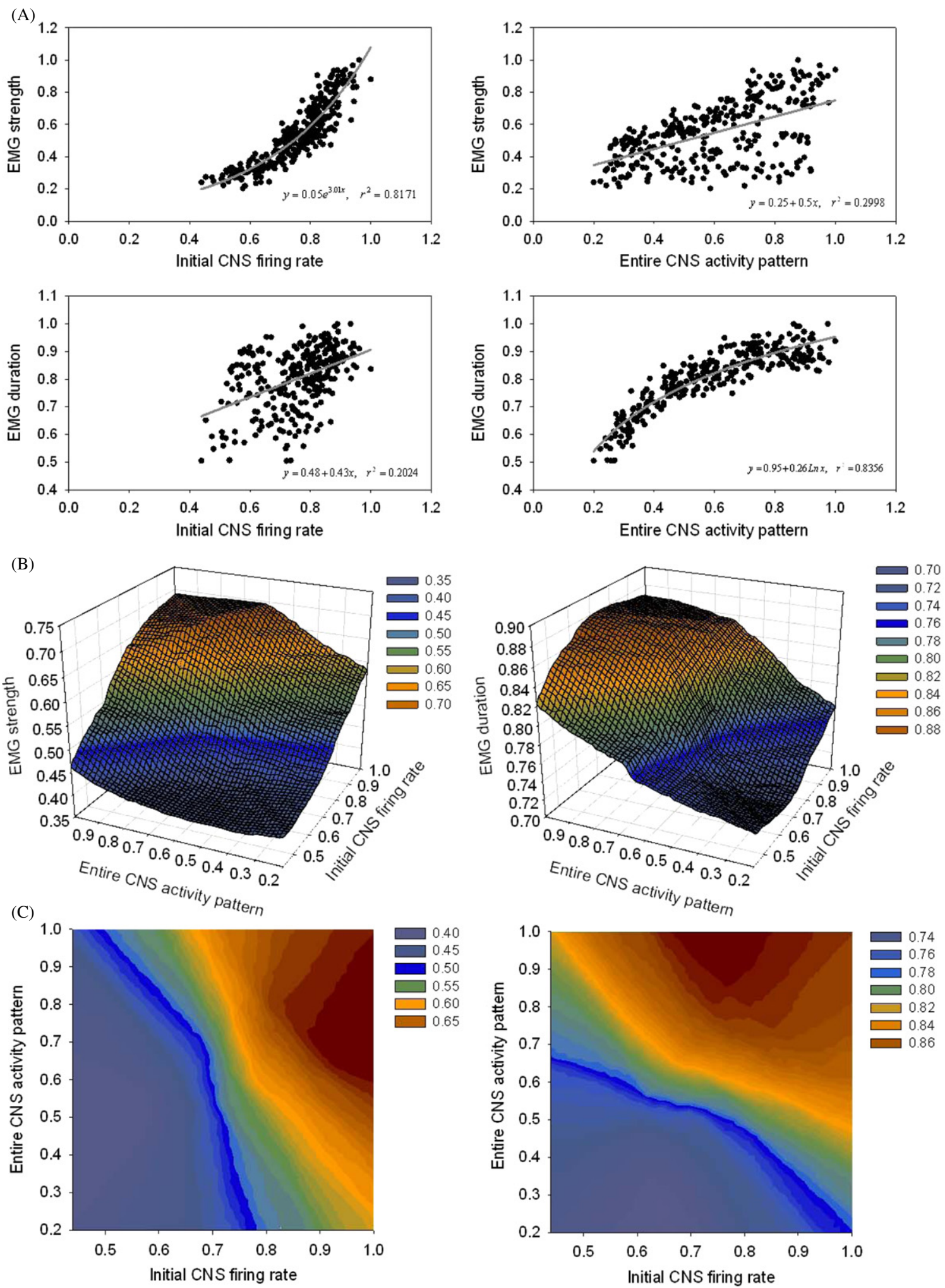

Figure 2. CNS activity originated ISMS and the resulting muscular responses. (A) Strength of muscular contractions as a function of early CNS neural activity (spiking rate during the first $15 \mathrm{~ms}$ ) affects the strength of muscle contractions exponentially (top left) and their duration linearly (bottom left). Strength of muscular contractions as a function of the duration of the entire CNS activity pattern affects the strength of muscle contractions linearly (top right) and their duration logarithmically (bottom right). (B) 3D representation of the combined effect of the two variables on the EMG activity. (C) Border line representation of the combined effect of the two variables on the EMG activity. All data are normalized; $n=5$ animals. 


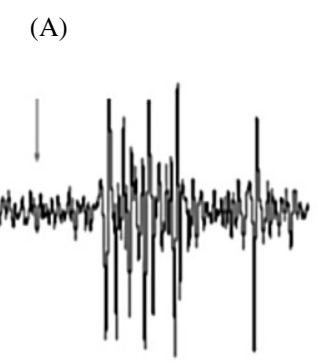

(C)

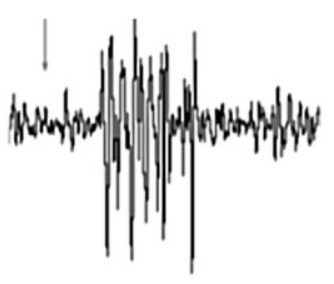

(A)

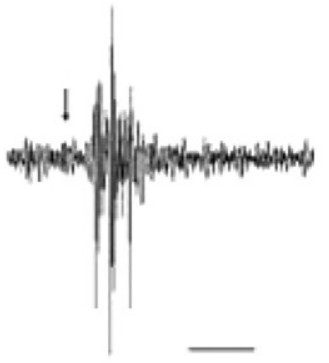

(D)

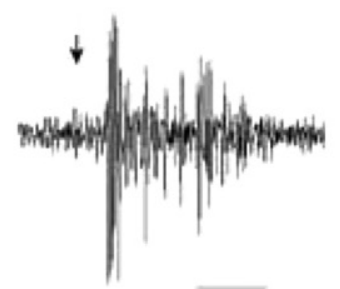

(B)

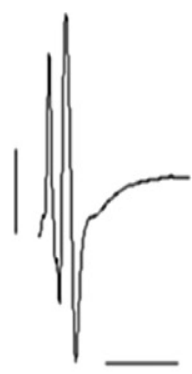

(D)

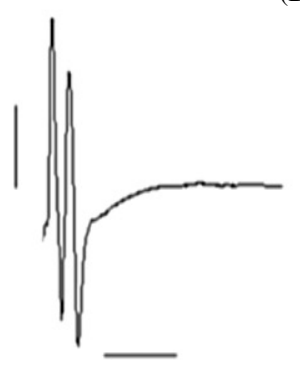

(B)

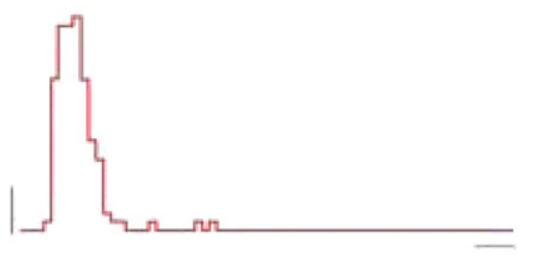

(E)

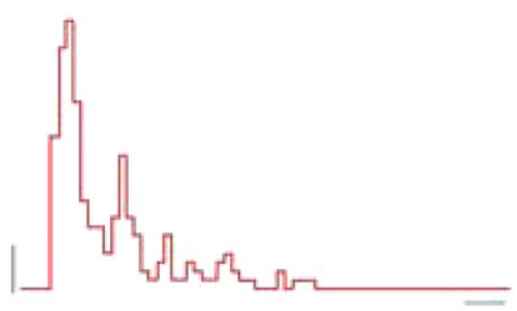

(E)

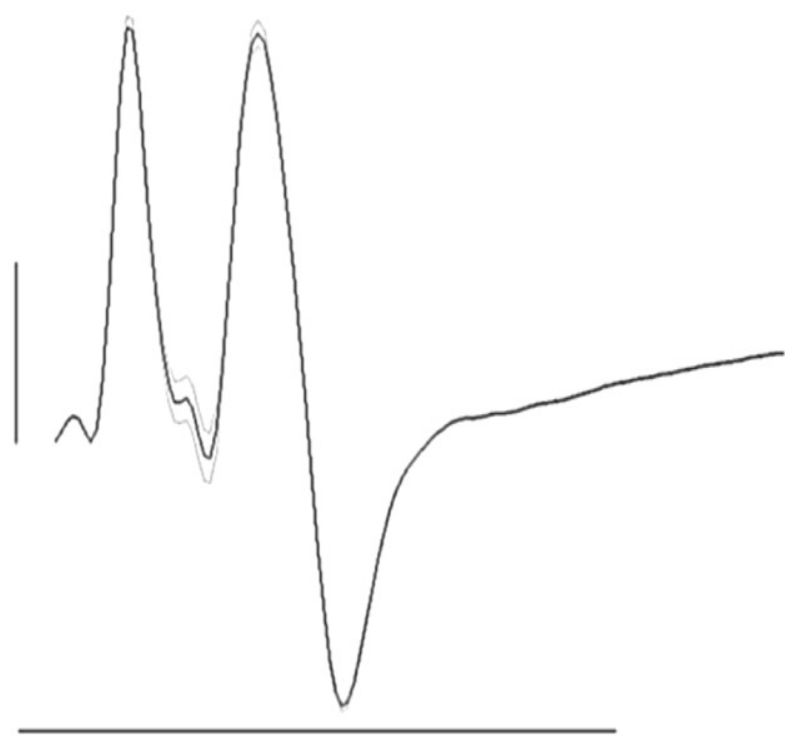

(C)

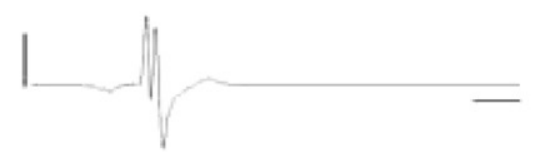

Figure 3. Top: reliability of the NSI procedure. ISMS triggered by the response of a Pr5 neuron to two $10 \mathrm{~ms}$ air-jet stimuli delivered to the same whisker (A) and (C) generate almost identical EMGs (B) and (D). The two Pr5 responses are slightly different due to the dynamics of the freely vibrating whisker. The mean EMG, built from the individual EMGs generated by 50 repetitions of the same air-jet stimulus, is shown in (E). The black line indicates the mean, and the gray line indicates the standard error of the mean. Note the very small variation of the EMGs. Bottom: neural patterns generated by 10 and $50 \mathrm{~ms}$ long air-jets delivered to a whisker. In rows (A) and (D) Pr5 recordings of the responses to two to three air-jet stimuli are shown. ISMS triggered by these recordings generated muscle contractions recorded by the EMGs (sensory feedback, (C) and (F)). (B) and (E) Summed peristimuli histograms of the responses to a series of 50 identical tactile stimuli ( $5 \mathrm{~ms}$ bin). In all cases, the horizontal bar corresponds to $10 \mathrm{~ms}$ and the vertical bar to $100 \mu \mathrm{V}$.

contractions, the strength of which depended linearly on the intensity of the injected current (figure 1, bottom right) and could be modulated by an external device. False movements, generated by non-desired (spontaneous) neural activity, were almost entirely absent.

To determine how the ISMS are related with CNS activity we studied the neural activity in Pr5 and the ISMSgenerated EMG in the muscles ( $n=5$ animals). By varying both the intensity and duration of the air-jet stimuli, we generated a wide spectrum of CNS neural activity that was recorded and converted to ISMS. The key variables were the initial CNS firing rate (the spiking rate of the overthreshold CNS activity during the first $15 \mathrm{~ms}$ ) and the duration of the entire activity pattern. These two variables were nonlinearly related to the strength and duration of the muscular responses as can be observed in figure 2. An early CNS spiking rate strongly affects the strength of muscle contractions and, faintly, their duration (exponentially in the first case and roughly linearly in the second, figure 2(A) left). Conversely, the duration of the entire CNS activity pattern strongly affects 

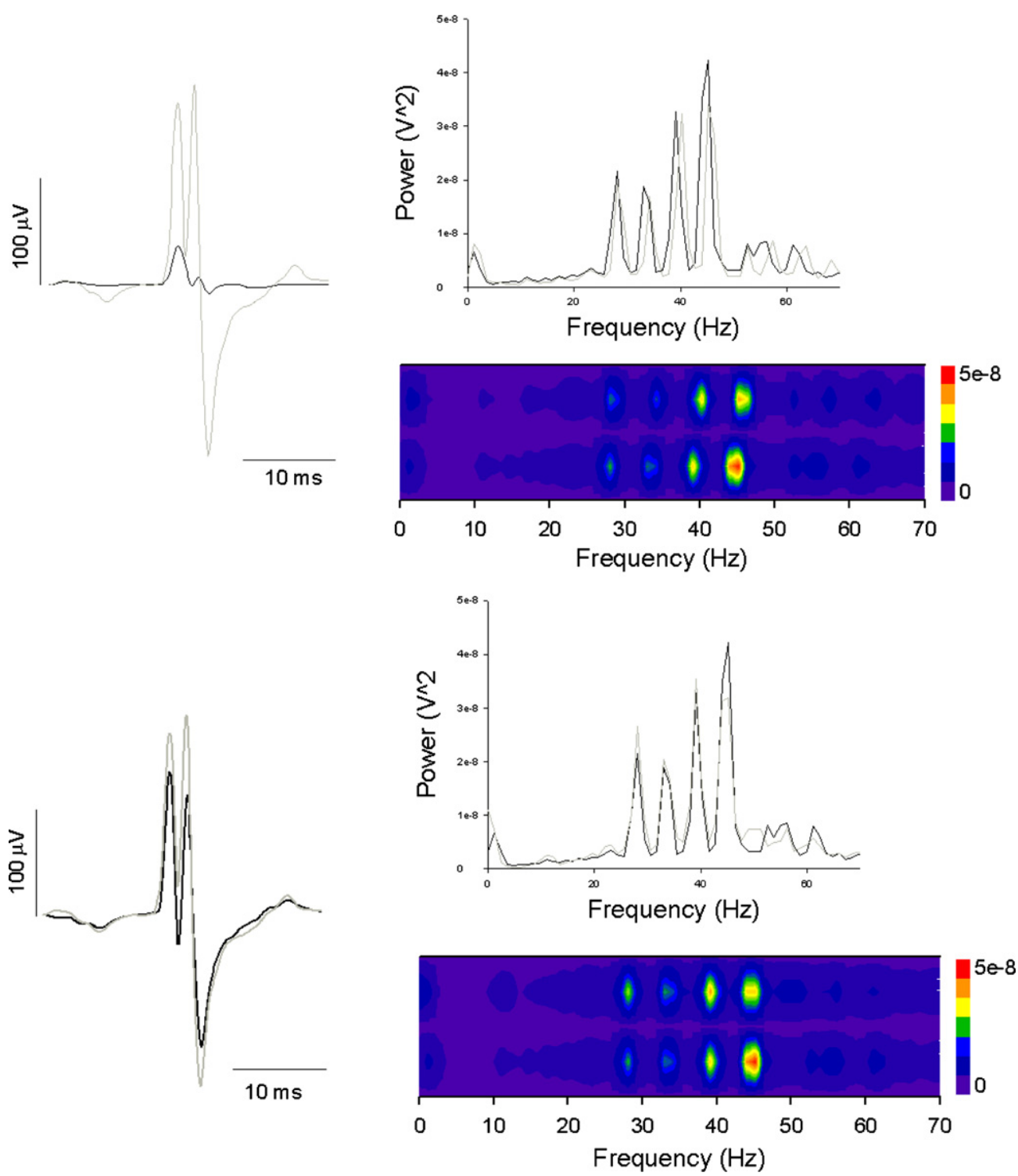

Figure 4. Top: mean EMGs obtained from the ISMS at two different depths of the spinal cord (left, gray and black line) using the same CNS neural pattern recordings (50 trials, $10 \mathrm{~ms}$ long stimuli delivered to the same vibrissa) and their power spectra (right). Bottom: the same, but obtained from the ISMS of a different animal.

the duration of the muscular responses and lessens their strength (linearly in the first case and logarithmically in the second, figure 2(A) right). In the same figure we show the combined nonlinear effect of the two variables on the strength (figures 2(B) and (C) left) and duration of the muscular response (figures 2(B) and (C) right).

The reliability of CNS-triggered fiber contractions was tested by analyzing the variability of the EMGs under repetition of the same CNS pattern. The process 'tactile stimulus $\Rightarrow \operatorname{Pr} 5$ response $\Rightarrow$ generation of ISMS $\Rightarrow$ fiber contraction' is not deterministic [22, 25]. However, the delivery of identical stimuli to a whisker resulted in very similar muscle fiber stimulation (see for example the mean and standard error of the EMGs in figure 3, top). ISMS and, consequently, muscle contractions depended on the duration of the CNS activity pattern even using the same time windows to trigger the ISMS (figure 3, bottom). Long CNS patterns displayed more likeness in their triggered ISMS.

We studied the robustness of the generation of precise movements by introducing variability in both the neural sampling and the spinal cord loci for the ISMS. EMG recordings show a clear dependence from the coordinates of the stimulation electrodes in the spinal cord (figure 4, top). Stimulation of the same spinal area in different animals evokes similar EMGs, both in shape and power spectrum, as proved by the comparison between animals (figure 4, bottom).

Coordinated movements resulted from the combinations of external stimuli with delays of $0-100 \mathrm{~ms}$ between them (figure 5, top). The precise timing of ISMS of spinal motor neurons triggered by the activity of the CNS provokes a synchronized contraction of muscle fibers with a consequent generation of coordinated movements (figure 5, bottom). 
(A)
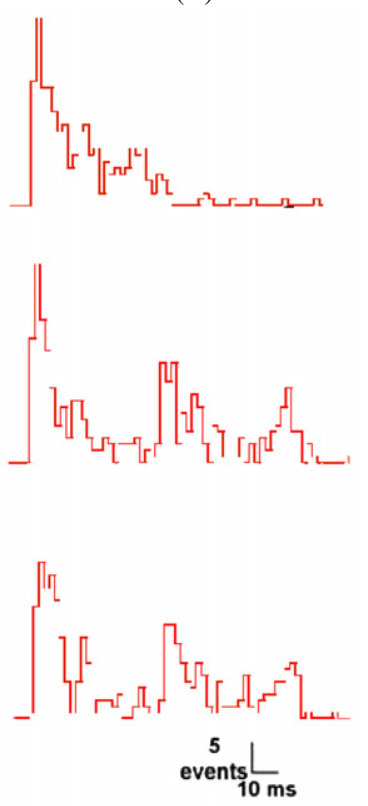

(A)

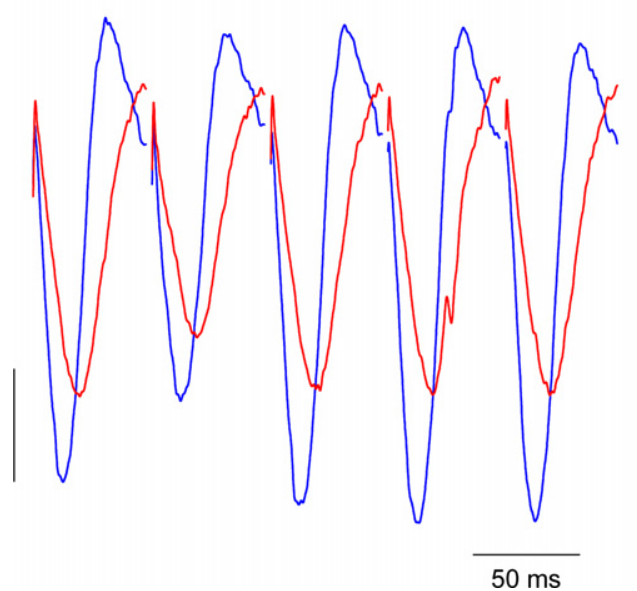

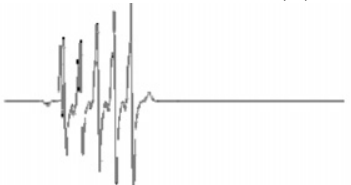
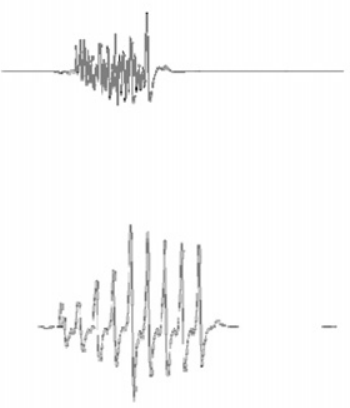

$1 \mathrm{mv}$ $20 \mathrm{~ms}$

(B)

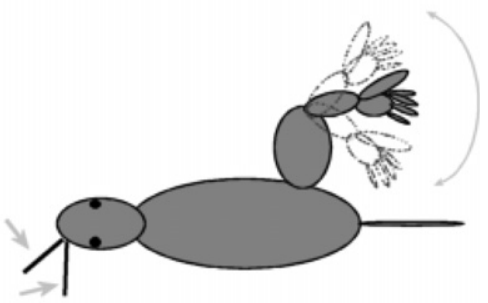

(C)

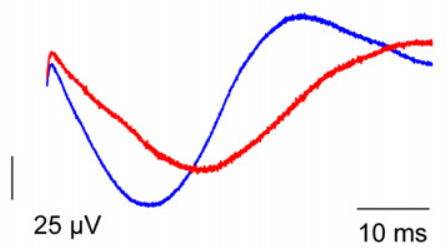

Figure 5. Top: generation of combined movements by means of two-whisker stimulations with $50 \mathrm{~ms}$ long tactile stimuli delivered with a delay of 0,10 and $50 \mathrm{~ms}$. Column (A) shows the summed peristimuli histograms of the combined Pr5 responses to a series of 50 identical tactile stimuli with the three delays (from top to bottom; $5 \mathrm{~ms}$ bin). ISMS triggered by these recordings generated combined fiber contractions recorded by EMGs (sensory feedback, column (B)). Bottom: simultaneous EMG recordings from soleus (red) and anterior tibialis (blue) muscles showing five cycles of coordinated contractions (A) generated by precise ISMS. ISMS were provoked from CNS recordings of the neural responses to air-puff stimulation of two different whiskers on the snout of the rat (B). Average EMGs from soleus (red) and tibialis anterior (blue) muscles obtained from 30 protraction-retraction cycles are shown in (C). The vertical bar corresponds to $25 \mu \mathrm{V}$.

\section{Discussion and conclusions}

In this paper, we have shown that selective ISMS of the post-lesioned motoneurons of a sectioned medulla could be achieved in a very precise and controlled manner using recordings of neural activity patterns from the CNS. Complex coordinated muscle movements can then be achieved by means of such recordings.

Precision in the generation of ISMS and coordinated muscle contraction is a necessary but not sufficient condition to achieve an acceptable mobility for spinal animals. It is necessary for synchronized motor behavior to be repeatable and robust for two reasons. The first is the lack of stability and reliability in long-term multiple single-unit recordings [9]. The second is the limited sampling of the desired neural activity due to the low number of recording electrodes we could implant in the CNS for the control of the neuroprostheses [11]. Therefore, the generation of correct muscle movements has to be independent on the variability of the neural sampling, and the generation of stable ISMS from arbitrary neural recordings is an imperative requirement for NSIs and their applications.

Accurate movements and coordination of muscle fiber contraction is coded by very specific neural activity patterns 
in the premotor and motor cortexes $[1,10]$. In the present experiments, robustness of the procedure was tested by introducing variability in the neural patterns used for the trigger of the ISMS. Reliability and robustness was proved by (i) analyzing similarities among ISMS triggered by non-identical air-puff stimuli, (ii) evaluating the efficiency of ISMS triggered by similar but different CNS neural patterns, and (iii) evaluating the repeatability of inter-animal stimulations.

Selective stimulation of the spinal motoneurons has been obtained taking into account the somatotopy of the spinal motoneurons and the trajectories of their efferent fibers in the rostrocaudal (alongside the spinal cord) and coronal (orthogonal to the spinal cord) planes. Medullar motoneurons are somatotopically distributed orthogonally to the medullar axis plane and along the longitudinal axis in a radius of less than $0.5 \mathrm{~mm}$. By avoiding the rostrocaudal placement of the electrodes, we avoided the stimulation of motoneurons dedicated to the same (or different but overlapping) region. By employing low intensity currents, we avoided the recruitment of a large amount of fibers that would result in an indirect stimulation of the muscles.

The finely coordinated muscle contractions we achieved, together with the sensory feedback from muscle fibers, could be used in future neuroprostheses/NISs to generate desired motor activity in paraplegic patients $[6,9,11,13,15]$. The sensory feedback from the CNS recordings from which we obtained the precise and coordinated muscle contractions could also be combined with fine recordings of muscle fiber activity to enhance the performance of the NISs. Such an approach could be built in a system with closed-loop control for ISMS to achieve functional electrical stimulation in people with spinal cord injuries (also in agreement with the evaluation of Mushahwar et al [30]). Our data on the use of sensory feedback and the fine control of peripheral muscles by means of ISMS are in agreement with experimental data from spinal decerebrated cats (i) on stimulation by unipolar microelectrodes to test ISMS for restoring locomotion after spinal cord injury [12], and (ii) on integration of interleaved ISMS in open- or closed-loop controllers with sensory feedback signals to modulate the amplitude of the applied stimulation [13].

Precise and selective CNS-generated ISMS may be used for many more therapeutic/clinical purposes. A clear example is spinal cord injury-induced spasticity. In their critical review, Elbasiouny et al [21] show that spinal cord injuryinduced spasticity depends on the increased excitability of spinal cord neurons (both motoneurons and interneurons) and the most promising approach they propose is spinal electrical stimulation to suppress it.

\section{Acknowledgments}

The authors would like to thank Celia Herrera-Rincon for histological studies and Vincent Jaskowski for the English language revision. This work has been supported by an IBERDROLA Grant (2004) to F Panetsos.

\section{References}

[1] Mason C and Kandel E R 1991 Principles of Neural Science ed E R Kandel, J H Schwartz and T M Jessell (New York: Elsevier) pp 421-39

[2] http://www.christopherreeve.org/site/c.mtKZKgMWKwG/ b.5193227/k.FC2/The_Costs_of_Living_ with_Spinal_Cord_Injury.htm

[3] http://www.campaignforcure.org/iccp/

[4] http://www.makoa.org/nscia/fact02.html

[5] Serruya M D, Hatsopoulos N G, Paninski L, Fellows M R and Donoghue J P 2002 Nature 416 141-2

[6] Hochberg L R, Serruya M D, Friehs G M, Mukand J A, Saleh M, Caplan A H, Branner A, Chen D, Penn R D and Donoghue J P 2006 Nature 442 164-71

[7] Lotze M, Laubis-Herrmann U, Topka H, Erb M and Grodd W 1999 Restor. Neurol. Neurosci. 14 183-7

[8] Frigon A and Rossignol S 2008 J. Physiol. 586 2927-45

[9] Hatsopoulos N G and Donoghue J P 2009 Annu. Rev. Neurosci. 32 249-66

[10] Moritz C T, Perlmutter S I and Fetz E E 2008 Nature $456639-42$

[11] Donoghue J P 2008 Neuron 60 511-21

[12] Saigal R, Renzi C and Mushahwar V K 2004 IEEE Trans. Neural. Syst. Rehabil. Eng. 12 430-40

[13] Lau B, Guevremont L and Mushahwar V K 2007 IEEE Trans. Neural. Syst. Rehabil. Eng. 15 273-85

[14] Mavoori J, Jackson A, Diorio C and Fetz E 2005 J. Neurosci. Methods 148 71-7

[15] Jackson A, Moritz C T, Mavoori J, Lucas T H and Fetz E E 2006 IEEE Trans. Neural. Syst. Rehabil. Eng. 14 187-90

[16] Jackson A, Mavoori J and Fetz E E 2006 Nature 444 56-60

[17] Fuentes R, Petersson P, Siesser W B, Caron M G and Nicolelis M A 2009 Science 323 1578-82

[18] Lavrov I, Courtine G, Dy C J, Van Den Brand R, Fong A J, Gerasimenko Y, Zhong H, Roy R R and Edgerton V R 2008 J. Neurosci. $287774-80$

[19] Gerasimenko M, Ichiyama R M, Lavrov I A, Courtine G, Cai L, Zhong H, Roy R R and Edgerton V R 2007 J. Neurophysiol. 98 2525-36

[20] Lebedev M A and Nicolelis M A 2006 Trends Neurosci. $9536-46$

[21] Elbasiouny S M, Moroz D, Bakr M M and Mushahwar V K 2010 Neurorehabil. Neural Repair 24 23-33

[22] Taylor D M, Helms Tillery S I and Schwartz A B 2002 Science 2961829

[23] Helms Tillery S I and Taylor D M 2004 Curr. Opin. Neurobiol. $14758-62$

[24] United Brachial Plexus Network info@ubpn.org http://www.ubpn.org

[25] Waite P M E and Tracey D J 1995 The Rat Nervous System ed G Paxinos (San Diego, CA: Academic) pp 705-24

[26] Diamond M E, von Heimendahl M, Knutsen P M, Kleinfeld D and Ahissar E 2008 Nat. Rev. Neurosci. 9 601-12

[27] Panetsos F and Sanchez-Jimenez A 2010 Neuroscience 169 893-905

[28] Enoka R M, Rankin L L, Stuart D G and Volz K A 1989 J. Physiol. 408 251-70

[29] Moreno A, Garcia-Gonzalez V, Sanchez-Jimenez A and Panetsos F 2005 Neuroreport 16 1569-73

[30] Mushahwar V K, Guevremont L and Saigal R 2006 IEEE Trans. Neural. Syst. Rehabil. Eng. 14 198-201 